\title{
Research on Fault Diagnosis Method Based on Grey Relation Degree
}

\author{
Chen Hongmin ${ }^{1, a}$, Zhang Minghu ${ }^{2, a}$, Li Hua ${ }^{1, b}$ and Mu Zhongguo ${ }^{2, b}$ \\ ${ }^{1}$ The Detachment of Warship Training, Dalian Naval Academy, Dalian, China \\ ${ }^{2}$ Dept. of Underwater Weaponry and Chemical Defense, Dalian Naval Academy, Dalian, China \\ azmhly20070618@163.com, bzmhly20040318@sina.com
}

Keywords: Grey relation analysis, grey relation degree, fault diagnosis, designs application.

\begin{abstract}
It is developed, applied the virtual instrument(VI) technology, grey system theory, and database, the fault diagnostic system for the shipboard weaponry equipment, in order to update entirely the means of the equipment testing. The modularization and universalization are proposed in its database-based design concept, realizing the design of the software and hardware. The ODBC technique is applied for the interconnection of databases to ensure the generality and flexibility of the system. The system mode the best of the VI platform and grey diagnosis method, broke through the conventional check diagnosis patterns for the warships weaponry equipment, solved the problems of state prediction and trouble-mode recognition of the warships weaponry equipment. It has been proved by the experiments that the system has merits both the high accuracy and economical practicability. Also it can reduce the application development cycle and cost.
\end{abstract}

\section{Introduction}

As a new practical system analysis technology, grey correlation analysis is the method of analyzing the correlation degree among the system factors. Its fundamental thought is comparing the similarity of the systematic counting array curves' geometry forms, and it describes the correlation degree among many factors, the more similar the geometry forms of array curves, the bigger the correlation degree between them is. Grey correlation analysis is not only one of the important achievements of gray system theory, but also the basics of grey forecast, grey decision-making, and grey control. It has offered the new thought and method for system analysis. And there are many projects using the method successfully. The description of the grey relation degree formulation has many types, e.g. trend correlation degree, T-type correlation degree, and absolute correlation degree etc.

The running weaponry equipment is a complex system, in which some information is known, the others is unknown, so we can call it a grey system. Fault diagnosis is a kind of method which uses the known information to identify the grey system's characteristic, state and tendency, moreover gives the forecast and decision-making.

\section{Diagnosis ratiocination of the grey correlation degree}

The diagnostic ratiocination program mainly consists three parts: the signal analyses and managements, the standard fault KBM (knowledge-base management), the grey correlation degree calculation and the fault model distinguish (see Fig.1). The signals analyses managements are the process of the mathematical analyses and transformations on the testing signals. The functions of $\mathrm{KBM}$ is mostly querying and updating the fault patterns. If there are no the corresponding fault pattern in established repository, the system will tell the user to input new standard pattern, and set up the homologous relationship with the currently tested objects, hence the new fault case has been added, and the fault repository will be extended at the same time. The grey correlation degree calculation is adapted to the conditions of the symptoms and causes of the faults are not very clear. Compared with the fuzzy ratiocination, the calculation of the grey correlation degree has no use for choosing the subject function and calculating on the fuzzy connection matrix. The dynamic database is used to record the results of the ratiocination and calculation processes. 


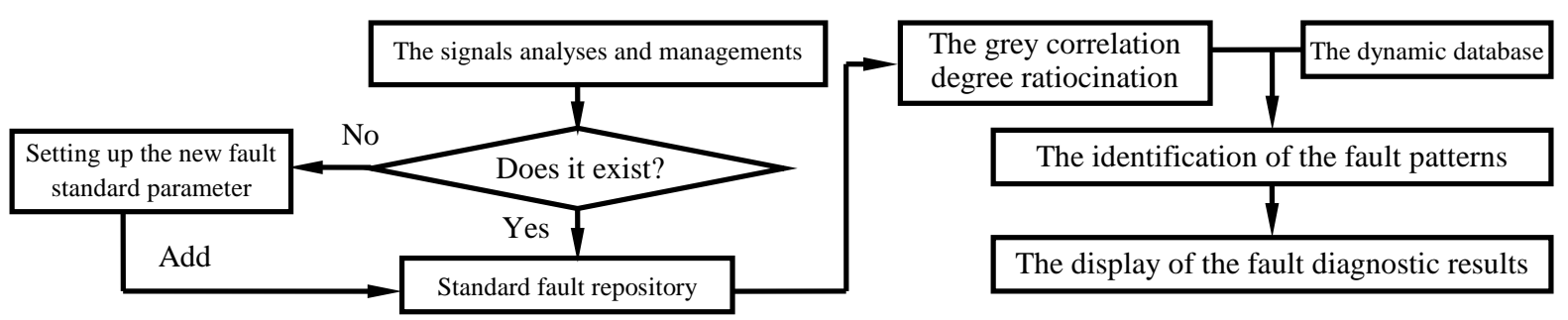

Fig. 1 The principle of fault diagnosis system

The grey correlation degree refers to the relevancy among the different objects, and it is used to confirm the practical pattern subjected to the certain standard one in the fault diagnoses for warship weaponry instrument. Suppose that in the fault diagnostic system, the number of the standard mode eigenvector is $\mathrm{m}$, that is to say, there are $\mathrm{m}$ kinds of the faults, if each standard mode eigenvector includes $n$ elements, the aggregate of the fault standard mode eigenvector is:

$$
X_{R}=\left\lfloor X_{r_{1}}(n), X_{r_{2}}(n), X_{r_{3}}(n), \cdots, X_{r_{m}}(n)\right\rfloor .
$$

If the eigenvector of the actual survey signals is:

$$
Y_{T}=\left[Y_{t}(1), Y_{t}(2), Y_{t}(3), \cdots, Y_{t}(n)\right] \text {. }
$$

The correlation degree coefficient between the actual eigenvector $Y_{T}$ and the standard eigenvector matrix $X_{R}$ at the point $\mathrm{K}$ is:

$$
\xi_{i j}(k)=\frac{\min _{i} \min _{j}\left|Y_{t}(j)-X_{r_{i}}(j)\right|+\rho \max _{i} \max _{j}\left|Y_{t}(j)-X_{r_{i}}(j)\right|}{\left|Y_{t}(j)-X_{r_{i}}(j)\right|+\rho \max _{i} \max _{j}\left|Y_{t}(j)-X_{r_{i}}(j)\right|} .
$$

In the above formula, $k=1,2, \cdots, n ; i=1,2, \cdots, m ; j=1,2, \cdots, n ; \rho$ is the distinguishing coefficient, $\rho$ $\in(0,1)$, commonly, it takes 0.5 .

Averaging the relation coefficients, the results are:

$$
r_{i}=\frac{1}{n} \sum_{k=1}^{n} W(k) \xi_{i j}(k) \text {. }
$$

Consequently, we will find the correlation degree sequence $[R]=\left[r_{1}, r_{2}, r_{3}, \cdots, r_{m}\right]$, in which $W(k)$ is the weight of every characteristic coefficient. When you choose the weights you should consider the effects of the characteristic coefficient on the operating condition of warship weaponry instrument, and the sum of them is 1 . If you choose the value ri to carry out the fault diagnostic identification, you should arrange the sequence according to the magnitude of the correlation degree between the actual mode eigenvector and the standard one, the standard fault mode which the correlation degree is the maximum is the proximal mode to the actual one at that time.

\section{Design on the fault diagnostic system of the warship weaponry equipment}

Technological Approach. In allusion to the electric condition and structure trait of the warship weaponry instrument, the fault diagnostic system adopts many advanced techniques, for example, the computer communication and control, virtual instrument(VI) testing, intelligent USB expanding, software module stack, multi-interface RAM and direct data read-in, DDS and neilsbed etc. It covers the main contents and demands of the technique regulations on warship weaponry instrument, may fulfill the tasks of the main existing examination and repair.

The system software adopts the modularization design, mainly including: the capability testing module, fault diagnostic module, expert-consultation module, virtual instrument testing module, control communication module and some suited periphery module. And the system can carry out the effective visitation to the power-setting and expert-ratiocination database by the ODBC technique.

The system hardware mainly includes the VI testing platform, master control circuitry or adapters and so on. When the VI testing platform uses the computer programme control, the mainboard CPU is driven through the USB extended interface, each of the functions modules is supported by the ratiocination software, and the capabilities or faults of warship weaponry equipment are thoroughly 
detected and analyzed.

Design of the software. Software plays an important role in impacting the system's performance, reliability, maintainability and expansibility.

Considered entirely for the incompatibility and traits of VI, the fault diagnostic system adopts manifold program languages that can drive the DLL (dynamic link libraries), in which mostly make use of Delphi and database. At the same time, the system also uses other languages, such as Visual Basic, Visual $\mathrm{C}++$, etc, to carry mixed programming through. So the system realizes the modularization and universalization design of the software, improves its maintainability and expansibility. The system software consists of the master control program module, and utility module library, mutual interface library, document module library, essential database, function module library and online help, etc (see Fig.2).

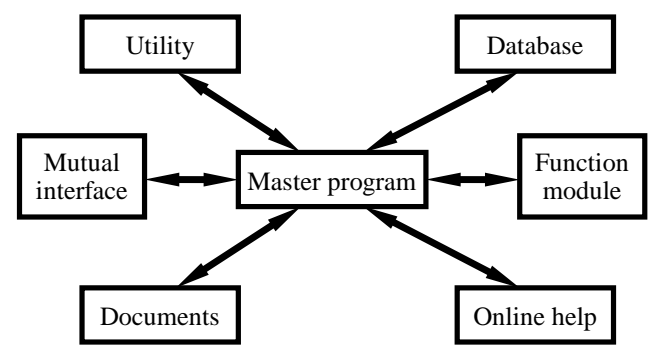

Fig.2 The composing of the system software

The master program is a control program of the high generality, which can be used to the man-machine conversation, interlinkage of database, flow control, instrument drive and documents handling, etc. But the given instrument or the functions operating can't emerge from the master control program, and the realization of all functions depends on transferring the function module programs and utility. The mutual interface library includes the VI softpanels, course hints and some essential operation interfaces. The utility library collects all kinds of the utilities necessary to the system. The essential database consists of the testing flow database, testing task database, testing instrument database, fault information library, expert information library and other necessary libraries. The function module library collects all of the function module programs, their name and path has been stored in the database as record, and they can be transferred by the master control program according to the practical need. The document library is used to store the system document files. The online help tells user how to use the system.

The finished main functions of the system program include the system setting-up, maintenance and management, to drive and use the different testing instrument, to use and manage the database resource, to implement the man-machine communication function, and so on. In which, the testing function is the most important one that the system will realize.

Design of the hardware. The design of the system hardware takes the thought of the function modularization, to improve the system flexibility, synthesis, adjustability, useability, reliability, maintainability, replantability, expansibility and practicability. The function modules of the hardware mainly include the USB interface module, definite quantity turned control isolation module, prepositive circuitry module and adapters, etc.

\section{The fault diagnosis examples}

Taking the familiar faults of the certain ship weaponry instrument as an example, we choose many same type instrument as stylebook, and simulate five kinds of the familiar faults, get the signal trait parameters at the fault mode respectively, and then set up the standard repository of the fault diagnosis. Simultaneously, we set the corresponding faults on the certain type ship weaponry instrument apart, measure the trait parameters of the different states, and calculate the correlation degree between every fault and its standard mode. The results indicate: when the correlation degree is assigned the maximum, the fault mode we set is nearly same as the standard one. Namely, the diagnostic result is almost consistent with the actual condition. 
When the same fault phenomenon is caused by the different reasons, the system can size the order according to the value of the correlation degree, and carry out the fault diagnoses step by step, till the fault has been eliminated.

\section{Conclusions}

In recent years, the VI has been widely used because of the superiority of the low cost and high capability. Applying VI technique and grey system theory to the fault diagnoses system of ship weaponry instrument, the two central tasks are the state-prediction and the fault mode identification. The results of the fault diagnosis examples indicate: the method is feasible; it can not only simplify the system hardware, but also make the signal analysis and result displaying more succinct; and the system organically combines the regular ratiocination with the grey correlation ratiocination, and makes the fault diagnosis and detection for the ship weaponry instrument more effective.

Now some concerned units have used the system, the results of the practical applications indicate: the system is of the advanced technique, reliable capability, handy operation, and the prodigious innovation and strong practicability, and overcomes the insurmountability difficulties which the faults of ship weaponry instrument are tested by the traditional method, shortens greatly the maintaining period, heightens the maintenance quality, and assures the speediness, veracity, reliability, maneuverability, maintainability and replantability of the examination and repair for ship weaponry instrument. Especially, the system is based on VI, so its use is wide, its operation is simple, and it has the traits of low price, credible performance, all-around function, convenient enlarging, and easy updating, and so on. Therefore, the fault diagnostic system has entirely innovated in the means of the performance testing, fault diagnosis and servicing suggestion for ship weaponry instrument. The

application of the VI not only decreases the development cost, improves the efficiency, but also greatly enhances the design agility, shortens the development cycle.

\section{Acknowledgements}

This work was financially supported in part by the national defense Pre-Research Found of China (41101050403).

\section{References}

[1] X. Zheng, F. Qian, Control and Instrument in Chemical Industry, No. 4 (2005), p. 1.

[2] M. Zhang, H. Wang, S. Lv, et al. Adv. Mater. Res. Vol. 108 (2010), p. 1515.

[3] V. Mane and V. Puri. Adv. Sci. Lett. Vol. 3(2010), p. 282.

[4] M. Zhang, D. Wang, Sh. Lv, et al. SENSOR LETTERS, Vol. 9, Issue 5(2011), p. 2104.

[5] Y. Han. Journal of Dalian Naval Academy, Vol. 36, No. 1 (2013), p. 19.

[6] S. Liu, H. Cai, Y. Yang, et al. Sys. Engineering - Theory \& Practive, No. 8 (2013), p. 2041.

[7] H. Zhao, J. Zhang, J. Zhang, Research and Exploration in Laboratory, No. 3(2014), p. 21.

[8] R. Sa, Zh.Hu, X. Zhang, et al.Machinery Design \& Manufacture, No. 11(2014), p. 61.

[9] X. Ren, X. Chen, Y. Yang, et al. Journal of Sichuan Ordnance, No. 5(2015), p. 86.

[10] H.Yao, J.Zhang, Y. Liu, et al. Power System Protection and Control, No. 9(2015), p. 115.

[11] Zh.Yang, L.Liang, X. Li, et al. Power System Technology, No. 6(2015), p. 1731.

[12] Y.Wang. Modern Defence Technology, No. 2(2015), p. 159. 